\title{
Les retouchoirs en matières dures animales : une comparaison entre Moustérien final et Uluzzien
}

\section{A comparison between Latest Mousterian and Uluzzian bone retouching tools}

\author{
Camille Anne Jéquier, Matteo Romandini, Marco Peresani* \\ Università degli Studi di Ferrara, Dipartimento di Biologia ed Evoluzione, Corso Ercole I d'Este, 32, 44121 Ferrara, Italie
}

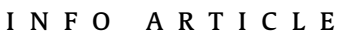

\section{Historique de l'article :}

Reçu le 21 octobre 2011

Accepté après révision $1^{\text {er }}$ décembre 2011

Disponible sur internet le 10 février 2012

Présenté par Yves Coppens

\section{Mots clés :}

Retouchoir

Os

Moustérien

Uluzzien

Italie

\section{R É S U M É}

Les retouchoirs en matières dures animales font l'objet de recherches toujours plus approfondies et ils s'avèrent très répandus, aussi bien géographiquement que chronologiquement et concernent notamment aussi bien le Moustérien final que l'Uluzzien en Italie. Afin de relever les éventuelles différences comportementales parmi les deux complexes culturels dans l'utilisation de ces outils, ce travail se base sur les éléments issus d'un site en Italie du Nord, la grotte de Fumane, qui a rendu plus de 150 pièces issues des deux technocomplexes à l'étude. Bien que les supports soient principalement en os, deux fragments de retouchoirs en andouiller de bois de cervidé sont à relever. Ils présentent une importante homogénéité, tant en ce qui concerne le spectre faunique (le cerf surtout), que les segments anatomiques (fémurs et tibias avant tout). Quatre types de stigmates ont été identifiés : les empreintes punctiformes, les empreintes linéaires, les stries et les cupules. Ils sont généralement regroupés en zones assez restreintes, avec jusqu'à trois superficies d'utilisation pour une diaphyse osseuse. Nous avons principalement relevé d'amples similitudes, bien que quelques différences existent entre les deux complexes culturels.

(C) 2012 Académie des sciences. Publié par Elsevier Masson SAS. Tous droits réservés.

\section{A B S T R A C T}

Retouching tools made of bone and other hard animal material have very broad chronological and geographical distribution throughout the Paleolithic period in Europe that also involves the Latest Mousterian and the Uluzzian in Italy. We investigated possible behavioural differences between these two cultural complexes in the use of these implements, based on the evidence from Fumane Cave, with its MP-EUP transitional sequence that has so far yielded more than 150 such artifacts. Although they are mostly made from bone shafts, a few remarkable examples of two fragments of cervid antler are also present. At a general level, these tools form an homogenous group: taxonomically, the bones fit with the faunal composition dominated by red deer and cervids, which were the most commonly hunted animals. The identified stigmata are punctiform impressions, linear impressions, striae and wells, usually grouped in small zones. Each retouching tool has up to three of these zones. Significant similarities in species, the skeletal part, and weight between the tools from the two cultural complexes have been detected, but also a difference due to the use of brown bear bones in the Uluzzian.

(c) 2012 Académie des sciences. Published by Elsevier Masson SAS. All rights reserved.

* Auteur correspondant.

Adresse e-mail : psm@unife.it (M. Peresani). 


\section{Abridged English version}

Although bone retouching tools comprise a specific class of the archaeological record known for more than a century, their function has been brought into question mainly during the past 20 years (Binford, 1981), that lead to detailed reconstructions. This body of research forms a basis for our study, which concerns over 150 fragmentary and complete bony retouching tools found in the Uppermost Mousterian (A5-A5+A6) and Uluzzian (A3-A4) layers at Fumane Cave in the North of Italy. This site contains a 60 ky-long sequence spanning the Middle Palaeolithic and the early Upper Palaeolithic, with large faunal and lithic assemblages. The final Mousterian layers record a prevalence of cervids over bovids and replacements in the LevalloisDiscoid lithic technology.

Because we consider the length of $5 \mathrm{~cm}$ used by Vincent (1993) for separating the analyzable from the not analyzable items too restrictive, a $1 \mathrm{~cm}$ wider range has been adopted in order to include other complete specimens. This resulted in the inclusion of 80 of 152 tools, with 7 of 11 in A3, 6 of 10 in A4, 21 of 48 in A5-A5+A6 and 46 of 83 in A6. Pieces fractured along the zone of use were excluded. The difference in the number of tools between the Uluzzian and the Uppermost Mousterian layers is due to differences in the degree of frequentation: the Uluzzian occupations were more sporadic than the earlier ones, which were subjected to more intense and repeated occupations.

Retouching tools were mostly made from the bone shafts of large mammals: red deer (Cervus elaphus) is the species most represented throughout the layers. The other specimens are mostly other cervids: giant-deer (Megaloceros sp.) and elk (Alces alces). Bone from bovids was also used, although rarely: Bos/Bison, ibex (Capra ibex) and chamois (Rupicapra rupicapra), as well as bone from bear (Ursus arctos). The femur was more commonly used than the tibia and metapodials. Fore limbs are rare, except metacarpals. Two fragments of cervid antler are also present. A high incidence of bones was fractured and modified, apparently during the recovery of the marrow. Our data also support comparison with the model advanced by Mozota Holgueras (2007a), that fracture of the long bone shafts was a deliberate procedure intended to recover fragments of appropriate size for retouching tools. The ranges of weight and size of the Fumane implements meet these assumptions: most range from 11 to 30 grams in weight, more clearly in the Mousterian than in the Uluzzian, which are almost $10 \mathrm{~cm}$ shorter in average than the former. On the contrary, breadths are comparable. Nevertheless, Mousterian tools have larger zones of use than those from the Uluzzians, but the relative breadth markedly prevails for the latter. The sizes of these zones have limited range, perhaps as a consequence of specific requirements for retouching. Four types of technical traces have been observed: punctual impressions, linear impressions, striae and notches. These stigmata can be found within one or several zones. Each tool usually shows a single zone, sometimes two or three.

Throughout the Fumane sequence, retouching tools share more similarities than differences in species, the skeletal part, and weight. One difference in the Uluzzian is the use of brown bear bones, known from other sites from the
Upper Palaeolithic. A comparison between the tools from Fumane and those from other sites underscores a similarity in the preferred use of some anatomical elements, such as the shafts extracted from the large herbivores. However, epiphyses are absent at Fumane, presumably due to the high degree of fragmentation or because they are underrepresented.

\section{Introduction}

Les retouchoirs en matières dures animales du Paléolithique sont connus depuis le début du $\mathrm{xx}^{\mathrm{e}}$ siècle; ils ont été décrits pour la première fois par Henri-Martin, 1906, dans le cadre de ses recherches à La Quina (1906). Par la suite, un débat nourri concernant notamment la fonction de ces fragments osseux utilisés a occupé les chercheurs pendant plusieurs décennies: ils auraient par exemple servi de dé à coudre (Bourlon, 1916) ou d'enclume, voire de perçoir (Gruet, 1947; Patou-Mathis et Schwab, 2002). Pour certains auteurs, principalement dans la première moitié du $\mathrm{xx}^{\mathrm{e}}$ siècle, les stigmates n'attestent que de traces de boucherie (cf. par exemple G. Chauvet, in Patou-Mathis et Schwab, 2002, concernant certains ossements mis au jour à La Quina). Leur mode d'utilisation fait encore l'objet de discussions: certains décrivent un geste de pression sur la matière à retoucher, d'autres préconisent la percussion. Cette question est cependant au centre de plusieurs publications (Armand et Delagnes, 1998 ; Bordes, 1961; Castel et al., 2003; Mallye et al., 2008; Mozota Holgueras, 2009 ; Vincent, 1993). Les auteurs penchent en général pour une utilisation en percussion lancée, bien que certains, tels que Bordes (1961) ou Castel et al. (2003) mentionnent que les stigmates caractéristiques peuvent être obtenus en effectuant une pression sur la pièce lithique à retoucher. Enfin, certains auteurs ont nié l'existence de ce type d'outil (Binford, 1981). Ces derniers n'ont cependant pas fait l'objet de recherches réellement approfondies avant les années 1990. De plus, les travaux dirigés par Patou-Mathis (2002) dans le cadre de la Commission de nomenclature sur l'industrie de l'os préhistorique, initiée par H.Camps-Fabrer, ont permis de rendre la problématique plus générale. Il en ressort que les supports utilisés sont aussi bien fabriqués à partir de bois de cervidé, d'os, que d'ivoire. Les segments anatomiques sélectionnés de préférence sont les diaphyses d'os longs, bien que les côtes et les épiphyses aient également servi. Quatre types de stigmates ont été identifiés : les empreintes punctiformes, les empreintes linéaires, les stries et les cupules, qui résultent de l'utilisation intensive des surfaces. Enfin, ces outils peu élaborés servent très probablement à la retouche des instruments lithiques. Les os utilisés proviennent en général d'animaux, bien que trois fragments de crâne humain aient été récemment publiés (Verna et d'Errico, 2011).

Présents dès le Paléolithique inférieur (Roberts et Parfitt, 1999), leur emploi se généralise au Paléolithique moyen. En France (Auguste, 2002; Patou-Mathis et Schwab, 2002), l'emploi de ces outils peu élaborés reste important au moment de la transition entre Paléolithique moyen et supérieur. Ils ont été retrouvés aussi bien en grotte que sur des sites de plein air, dans des gisements contenant des industries très variées (Levallois, 
Discoïde, Aurignacien, etc.). Toutefois, les études sur certaines industries de transition, telles que le Châtelperronien, le Néronien (Slimak, 2007) ou le Szélétien (Oliva, 1995 ; Simán, 1995), ne se sont pas encore tournées vers l'utilisation ou non des retouchoirs et sur leur éventuelle intégration au sein de l'industrie sur matières dures animales. À ce propos, il faut aussi souligner que, dans la zone centrale du Nord méditerranéen, peu de chercheurs se sont penchés sur cette problématique, exception faite des analyses technologiques et fonctionnelles de l'industrie en matières dures animales de la Grotta della Cala, de la Grotta del Cavallo et de la Grotta di Castelcivita (D'Errico et al., sous presse). En effet, les études sur l'utilisation des matières dures animales comme supports de retouchoirs peuvent apporter une contribution non négligeable au débat toujours vif qui tourne autour des dynamiques bioculturelles et à l'attribution de l'origine de l'exploitation formelle de l'os, de l'ivoire ou du bois de cervidé à l'Homme moderne ou à Néandertal. Les recherches conduites sur la grotte de Fumane se placent dans ce cadre et apportent une contribution significative sur l'emploi de ces outils et sur leur variabilité.

\section{Présentation de la grotte de Fumane}

La grotte de Fumane présente en effet une stratigraphie de premier ordre qui couvre une période allant d'au moins 90000 ans à la phase ancienne du Paléolithique supérieur. Située à environ $350 \mathrm{~m}$ d'altitude, dans la province de Vérone, au pied des Monti Lessini, la Grotte a fait pour la première fois l'objet d'une fouille en 1964, à la suite de l'élargissement de la route qui la longe. Dans un premier temps, seules les couches inférieures ont été reconnues puis, en 1988, un système de cavités et de galeries est mis au jour et livre un riche ensemble du Moustérien final à l'Aurignacien. Aujourd'hui, le site est toujours fouillé et présente une stratigraphie de plus de $12 \mathrm{~m}$ de puissance. La Grotte de Fumane a fait l'objet de nombreuses publications qui ont couvert les principaux aspects de l'occupation humaine à la fin du Moustérien et durant l'Aurignacien (Broglio et Dalmeri, 2005 ; Broglio et al., 2006 ; Cassoli et Tagliacozzo, 1994 ; Peresani et al., 2008, 2011b); nous n'y reviendrons donc pas. Précisons toutefois que les pièces dont il sera question ici proviennent du complexe de couches A5-A6 pour le Moustérien final et A3 et A4 pour les niveaux uluzziens.

\subsection{La séquence stratigraphique A3-A6: généralités}

Ces couches ont fait l'objet de fouilles extensives dans l'aire située devant l'actuelle limite du porche de la grotte, ainsi qu'à son entrée. La superficie de fouille a été quadrillée en carrés de $33 \times 33 \mathrm{~cm}$. Le matériel a été récolté lors de l'extraction à proprement parler (os et industrie lithique), puis par tamisage à l'eau.

Les unités sédimentaires du complexe A5-A6sont déposées au-dessus de l'A7, une couche complètement stérile du point de vue de l'occupation anthropique primaire. Elles forment un ensemble sub-horizontal de quasiment $20 \mathrm{~cm}$ d'épaisseur. Du fond de la cavité en direction de l'extérieur, les pierres sont progressivement remplacées par des sables éoliens, jusqu'à leur complète disparition. Les unités principales sont l'A6, une couche caractérisée par une grande densité de matières organiques, de charbons, de faune et d'artefacts lithiques; l'A5+A6, plus riche en pierres à l'entrée de la grotte qu'au-delà de l'actuelle ligne de pluie ; enfin, l'A5, un horizon présentant des structures de combustion ainsi que des déchets, mis au jour dans la partie ouest de la surface fouillée. L'A6 est subdivisée en trois zones : la zone ouest, où se trouvent plus de vingt structures de combustion et accumulations localisées de résidus de combustion; une aire de rejet caractérisée par un ensemble de matériels brûlés ou chauffés et, enfin, une grande superficie située à proximité de la paroi opposée contenant un grand nombre de déchets de production lithique, d'outils et d'os modifiés par l'homme. Les occupations anthropiques sont moins intenses en $A 5$ et A5+A6 (Peresani et al., 2011b). Les datations au radiocarbone ont donné $40150 \pm 350 \mathrm{BP}$ et $41650 \pm 650 \mathrm{BP}$ pour le niveau A5 et $40460 \pm 360 \mathrm{BP}$ pour A5+A6 (Higham et al., 2009). L'industrie lithique Levallois a pour objectif l'obtention de lames à bords parallèles ou convergents (Peresani, 2012). La modalité unidirectionnelle récurrente a été préférée jusqu'à la phase terminale de la séquence de réduction, où elle est remplacée par une modalité centripète. La retouche est principalement utilisée pour façonner des racloirs et des pointes, en proportions variables entre A6 et A5, au détriment des encoches et des denticulés. Ces derniers s'avèrent rares; il en va de même pour les éclats simples amincis, ainsi que pour les autres types d'outils. Les racloirs simples prédominent sur les autres, notamment sur ceux à retouche alterne ou marginale.

Les couches uluzziennes, A3 et A4, sont principalement constituées de pierres fragmentées par la gélifraction. Elles contiennent également du sable et des limons éoliens en quantité variable, plus abondants à l'entrée de la grotte et s'amenuisant à l'intérieur. Des structures de combustion, comprenant des foyers et une zone de déchets comprenant des produits secondaires taillés, des charbons et quelques os ont été mises au jour (Peresani, 2008). L'Uluzzien coïncide avec plusieurs innovations concernant les procédures technologiques, comme la production de lames et de lamelles, ainsi que l'utilisation d'une nouvelle gamme d'outils. La méthode Levallois est présente surtout dans la phase initiale (c'est-à-dire la couche A4), bien que de modalité centripète plutôt qu'unidirectionnelle récurrente, et disparaît par la suite. Les autres éclats ont été produits suivant des méthodes variées, en vue d'extraire des éclats polygonaux ou rectangulaires à dos épais opposé au bord coupant (Peresani, 2012). Les racloirs sont les principaux outils retouchés, surtout dans la première phase de fréquentation, avec des pièces à dos et des pièces esquillées. La proportion de ces dernières augmente dans l'A3, en parallèle avec l'apparition de quelques outils en matières dures animales, comme un perçoir et un coin, en cours d'étude.

\subsection{Quelques données archéozoologiques}

L'analyse de l'assemblage faunique du Moustérien final et de l'Uluzzien témoigne d'un riche assemblage d'ongulés, de carnivores et d'oiseaux, appartenant à des environnements et des climats variés. Les espèces les plus chassées 
sont, en ordre décroissant: le cerf (Cervus elaphus), le bouquetin (Capra ibex), l'élan (Alces alces) et, dans une moindre mesure, le chamois (Rupicapra rupicapra), le chevreuil (Capreolus capreolus), le mégacéros (Megaloceros giganteus), un boviné (Bos/Bison) et le rhinocéros laineux (Coelodonta antiquitatis). Parmi les carnivores, les restes de renard prévalent nettement sur ceux de loup (Lupus lupus), de hyène (Crocuta crocuta), d'ursidés (Ursus arctos, Ursus spelaeus), de mustélidés (Mustela erminea, Mustela nivalis, Gulo gulo) et des félins (Lynx lynx, Panthera pardus). Les vestiges de Lepus sp. et de rongeurs, représentés uniquement par la marmotte, sont extrêmement rares, tandis que les restes d'oiseaux sont nombreux et portent des traces anthropiques (Peresani et al., 2011a ; Tagliacozzo et al., à paraître).

Des modifications anthropiques ont été reconnues aussi bien sur des spécimens identifiés, que sur des fragments indéterminables. En ce qui concerne les éléments reconnaissables, les traces anthropiques comprennent les stries attribuables à différentes activités de boucherie sur presque tous les ongulés issus des unités étudiées. De nombreux cônes de percussion ont été reconnus sur les éléments indéterminables, ce qui a permis d'émettre l'hypothèse de la fragmentation des os, afin d'obtenir la moelle osseuse. Il semble que le haut degré de fragmentation du matériel osseux soit surtout à attribuer aux activités de boucherie, à la combustion et à des processus naturels. En effet, les traces laissées par les rongeurs sont presque complètement absentes et celles typiques des carnivores s'avèrent relativement rares. Il est ainsi possible de suggérer que ces derniers n'ont pas joué un rôle prépondérant, lors de l'accumulation des restes osseux à Fumane. Il est cependant probable que la hyène ait fréquenté sporadiquement la grotte, en alternance avec l'homme, puisque quelques ossements de grande dimension ont été digérés.

Enfin, des traces de boucherie identifiées sur quelques pièces attribuables à des carnivores (partie distale des os longs) indiquent une attention particulière portée à l'acquisition de la fourrure (Peresani et al., 2011b; Romandini, 2007-2008; Tagliacozzo et al., à paraître).

Le spectre faunique, ainsi que des comparaisons quantitatives, ont permis de souligner que peu de modifications écologiques et économiques existent entre les complexes culturels. Les niveaux uluzziens attestent une tendance à l'élargissement du spectre faunique chassé, en ce qui concerne les carnivores notamment (renard, loup, ours brun), au contraire des couches moustériennes où seul l'ours brun porte des traces dues à la consommation.

\section{Objectifs, matériels et méthodes}

Ce travail sur les retouchoirs en matières dures animales a pour but de mieux caractériser ces objets peu élaborés, de déterminer si certains traits se différencient entre les deux groupes culturels et, enfin, d'apporter de nouveaux éléments d'étude sur ces outils en matières dures animales. Les retouchoirs de ces niveaux constitueront un point de référence utile pour comparer les couches aurignaciennes aux couches discoïdes sous-jacentes, encore en cours de fouille. Il faut mentionner également la présence de deux retouchoirs lithiques, obtenus de façon opportuniste sur des éclats corticaux, et de quelques galets plats en serpentine et en calcaire, mis au jour dans les niveaux moustériens. Les stigmates caractéristiques de la percussion sont aisément reconnaissables. L'étude de ces objets est en cours et les résultats seront exposés lors de futurs travaux.

Les retouchoirs ont été regroupés en quatre niveaux stratigraphiques, y compris leurs diverses sous-unités relatives. L'unité $\mathrm{A} 5$ à été incluse à l'A5+A6 (à lire dorénavant $A 5-A 5+A 6)$. Les pièces ont été reconnues la plupart du temps à l'œil nu, à la lumière naturelle et artificielle, à partir de deux corpus archéologiques distincts. Le premier a, en effet, été prélevé lors de la fouille, avec enregistrement de chaque pièce de plus de $5 \mathrm{~cm}$. Le second provient du tamisage à eau, afin de récupérer les éléments restants. Dans de rares cas, nous avons utilisé une loupe binoculaire, voire un microscope. Ce dernier, un Leica S6D Green Ough à agrandissement de 10 à 64 fois, nous a toutefois été utile pour contrôler certaines pièces et mieux définir les stigmates. Nous avons scanné, à l'aide d'un scanner Epson Perfection V350 photo, tous les retouchoirs et avons travaillé les photographies sur Adobe Photoshop CS4 pour Mac.

Plusieurs types de mesures ont été calculés sur les retouchoirs. La longueur et la largeur maximales de chaque pièce ont été prises à l'aide d'un pied à coulisse, en millimètres ; il en va de même pour les zones d'utilisation. En outre, une balance a servi pour peser les fragments, au décigramme près.

Ont été considérés comme entiers les retouchoirs qui mesuraient $4 \mathrm{~cm}$ et plus, à l'exception des éléments qui comportaient une fracture évidente sur la zone d'utilisation. Ces derniers ont été écartés de l'étude puisque incomplets.

Vincent (1993) soutient que les retouchoirs doivent mesurer au moins $5 \mathrm{~cm}$ de long pour être fonctionnels. Nous trouvons cette distinction trop réductrice. En effet, il est difficile d'estimer la longueur originelle du support. Certaines pièces portent les stigmates de fractures produites durant la phase d'utilisation ou dues à des phénomènes postdépositionnels. D’autres éléments, qui mesurent parfois moins de $2 \mathrm{~cm}$ et portent clairement une fracture sur la zone d'utilisation, démontrent que le degré de fracturation peut être élevé.

En ce qui concerne la typologie des stigmates techniques, nous avons adopté la nomenclature proposée par Mozota Holgueras dans son mémoire et publiée par la suite dans un article (Mozota Holgueras, 2007a,b).

\section{Résultats}

La conservation des restes est souvent excellente (Fig. 1), dans la mesure où les traces anthropiques se lisent facilement et où les altérations naturelles n'empêchent généralement pas l'analyse des stigmates de boucherie ou de technologie. Les surfaces osseuses sont généralement bien conservées, bien que l'on observe des dépressions et des sillons laissés par les racines, ainsi que des microfissures probablement attribuables à des phénomènes de weathering et/ou des weathering cracks. Les traces de manganèse, de concrétion, de piétinement et d'exfoliation sont présentes mais peu fréquentes. 
a)

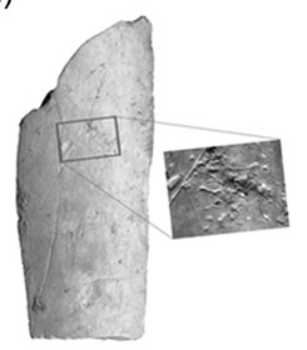

b)

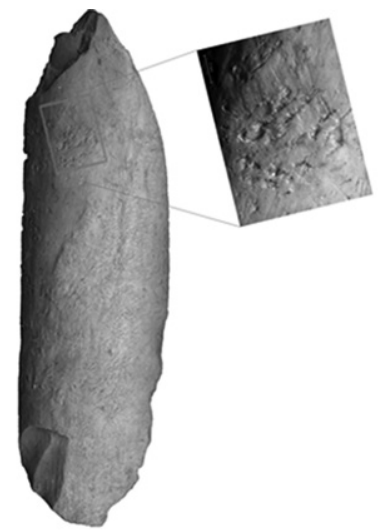

d)

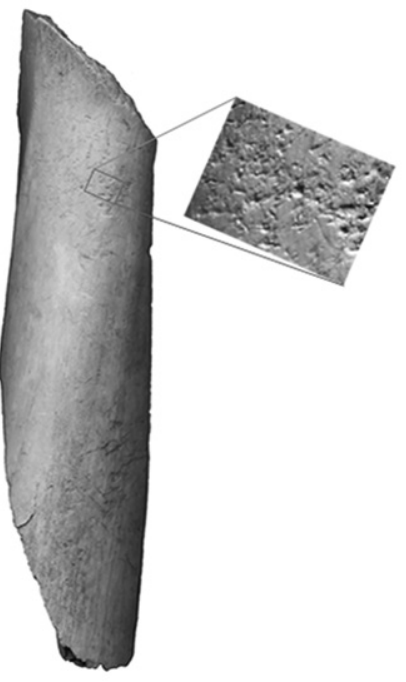

Fig. 1. Retouchoirs : a : unité A4, impressions punctiformes; b: unité $A 5+A 6$, impressions punctiformes et stries de raclage sous-jacentes; $c$ : unité $A 6$, impressions punctiformes et linéaires; $\mathrm{d}$ : unité $\mathrm{A6}$, intersection de deux zones à impressions punctiformes.

Fig. 1. Retouchers: a: unit $A 4$, punctiform impressions; b: unit $A 5+A 6$, punctiform impressions on scraping strias; $c$ : unit $A 6$, punctiform and linear impressions; d: unit A6, intersection with two use zones, both punctiform impressions.

Nous avons individualisé 152 retouchoirs provenant des quatre couches en question. Comme mentionné précédemment, les éléments de moins de $4 \mathrm{~cm}$ ou portant une fracture au sein de la zone d'utilisation ont été éliminés. Nous avons donc considéré 80 retouchoirs entiers (Fig. 2).

Il faut relever que la grande différence numérique entre les couches uluzziennes et les niveaux moustériens est à attribuer aux fréquentations intenses et répétées de la grotte durant la période plus ancienne (Peresani et al., 2011b; Peresani, 2008).

\subsection{Détermination taxinomique et anatomique}

Comme le montre le Tableau 1, le cerf représente la grande majorité des espèces déterminées, aussi bien dans les couches uluzziennes que dans celles du Moustérien final. Toutefois, les retouchoirs sont également souvent présents sur les taxa de gros module, tels que le mégacéros ou les bovidés. Le spectre faunique associé aux retouchoirs respecte bien celui qui a été mis en évidence lors des

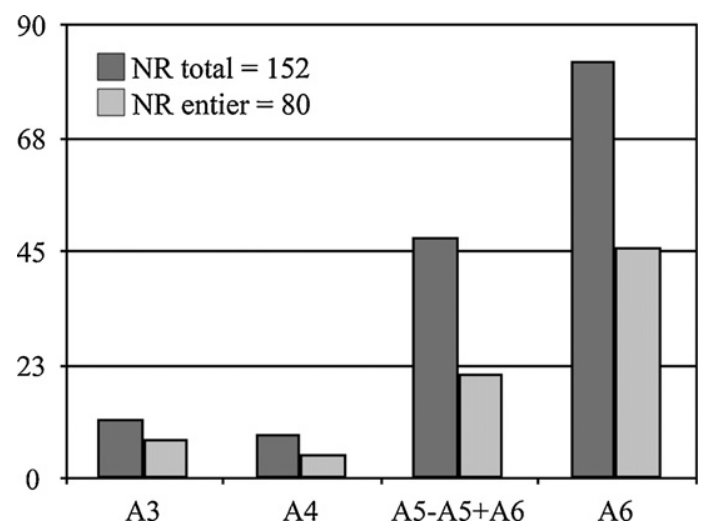

Fig. 2. Nombre de retouchoirs avec et sans les fragments par unité stratigraphique.

Fig. 2. Number of retouchers with and without the fragments in each stratigraphic unit. 
Tableau 1

Attribution taxinomique des retouchoirs.

Table 1 Taxonomical attribution of the retouchers.

\begin{tabular}{|c|c|c|c|c|c|c|c|c|}
\hline \multirow[t]{2}{*}{ Taxa/unité } & \multicolumn{2}{|l|}{ A3 } & \multicolumn{2}{|l|}{ A4 } & \multicolumn{2}{|c|}{$\mathrm{A} 5-\mathrm{A} 5+\mathrm{A} 6$} & \multicolumn{2}{|l|}{ A6 } \\
\hline & NR & $\%$ & NR & $\%$ & NR & $\%$ & NR & $\%$ \\
\hline Ursus arctos & & & 1 & & & & & \\
\hline Cervus elaphus & 7 & & 2 & & 12 & 57,1 & 28 & 60,9 \\
\hline Cervus-Megaloceros & & & & & 2 & 9,5 & 5 & 10,9 \\
\hline Cervus-Alces-Megaloceros & & & & & & & 2 & 4,3 \\
\hline Megaloceros-Alces & & & & & 1 & 4,8 & 2 & 4,3 \\
\hline Megaloceros-Alces-Bos/Bison & & & & & & & 1 & 2,2 \\
\hline Megaloceros-Bos/Bison & & & 1 & & 2 & 9,5 & & \\
\hline Cervidae & & & & & 1 & 4,8 & & \\
\hline Bos/Bison & & & & & 1 & 4,8 & 1 & 2,2 \\
\hline Rupicapra rupicapra & & & & & & & 1 & 2,2 \\
\hline Capra ibex & & & & & & & 1 & 2,2 \\
\hline Ungulata & & & & & & & 2 & 4,3 \\
\hline Ind. grosse taille & 1 & & 1 & & 2 & 9,5 & 3 & 6,5 \\
\hline Total & 8 & & 5 & & 21 & 100,0 & 46 & 100,0 \\
\hline
\end{tabular}

analyses archéozoologiques, mais quelques pièces méritent d'être mentionnées. En effet, les couches uluzziennes, en particulier l'A4, sont les seules à contenir des restes d'ours: un fragment de fémur d'ours brun a servi de support de retouchoir. L'utilisation de fragments osseux d'ursidés n'est pas inconnue durant le Paléolithique moyen : en effet, Valensi et Psathi (2004) mentionnent la récupération d'une diaphyse d'ulna comme retouchoir. En revanche, les couches moustériennes contiennent quelques pièces réalisées sur des fragments appartenant à des animaux de petite à moyenne taille, comme le chamois et le bouquetin.

D'une manière générale, toutefois, les artisans de l'industrie lithique ont préféré utiliser des fragments provenant d'animaux robustes, ayant une plus forte résistance aux coups donnés lors de la retouche.

Pour appuyer cette hypothèse, les segments anatomiques choisis en premier lieu montrent des caractéristiques de robustesse et de résistance aux chocs importants. En effet, les fémurs, tibias et métapodes ont été principalement sélectionnés, probablement en relation avec la section relativement épaisse des fragments (Tableau 2). Les différentes couches montrent, comme pour la détermination taxinomique, une bonne homogénéité des pièces recherchées : les fémurs avant tout (sauf pour l'A5-A5+A6, qui contient trois fois plus de tibias que de fémurs), les tibias et les métapodes, principalement les métacarpes. Les fragments d'humérus et de radius ont été plus rarement utilisés. Le fragment de mandibule, de gros module, représente également une pièce peu documentée.

\subsection{Acquisition des retouchoirs}

Les diaphyses d'os longs de cervidés et de bovidés portent fréquemment des traces de boucherie ou de fracturation, indiquant ainsi que la matière première était disponible en grande quantité à l'intérieur de la grotte. En ce qui concerne les seuls retouchoirs, la Fig. 3 montre les différentes actions anthropiques qui sont liées à l'enlèvement des tissus carnés et à la fragmentation des diaphyses. Les points d'impacts sont relativement fréquents et indiquent la récupération de la moelle osseuse, ce qui permettait dans le même temps de fournir des supports aux dimensions adéquates pour remplir leur rôle de retouchoir.

Les mesures des retouchoirs ne sont pas définies avec précision. Toutefois, ils ne doivent être, ni trop petits, ni trop légers. Vincent proposait une longueur minimale de $5 \mathrm{~cm}$, bien que cela ne soit pas l'unique dimension à prendre en compte. En ce qui concerne la grotte de Fumane, les dimensions des retouchoirs sont plus importantes dans les couches moustériennes que uluzziennes, comme en témoigne le Tableau 3. Toutefois, les largeurs occupent le

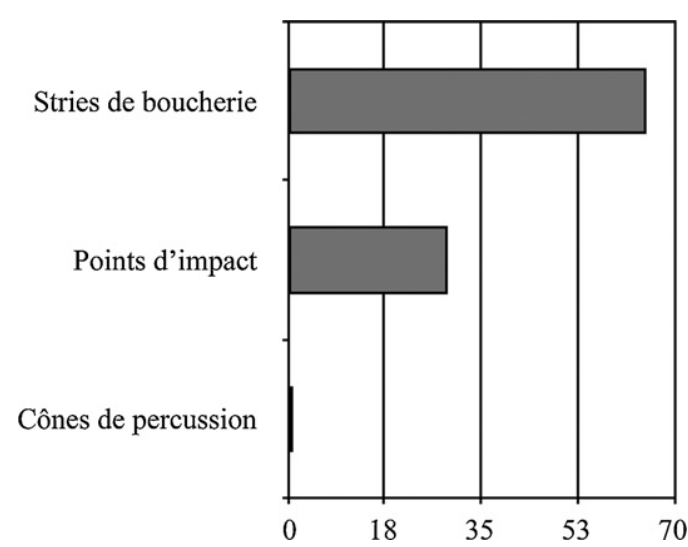

Fig. 3. Typologie des altérations anthropiques relevées sur les pièces entières.

Fig. 3. Types of anthropic modifications observed on the complete artifacts. 
Tableau 2

Détermination du segment anatomique des retouchoirs.

Table 2 Determination of the anatomical part used as retoucher.

\begin{tabular}{|c|c|c|c|c|c|c|c|c|}
\hline \multirow[t]{2}{*}{ Porlion atomique/unité } & \multicolumn{2}{|l|}{ A3 } & \multicolumn{2}{|l|}{ A4 } & \multicolumn{2}{|c|}{$A 5-A 5+A 6$} & \multicolumn{2}{|l|}{ A6 } \\
\hline & NR & $\%$ & NR & $\%$ & NR & $\%$ & NR & $\%$ \\
\hline Humérus & 1 & & & & 1 & 4,8 & 4 & 8,7 \\
\hline Radius & & & 1 & & 3 & 14,3 & 4 & 8,7 \\
\hline Métacarpe & 1 & & & & 2 & 9,5 & 6 & 13,0 \\
\hline Fémur & 2 & & 2 & & 3 & 14,3 & 14 & 30,4 \\
\hline Tibia & 2 & & 1 & & 9 & 42,9 & 12 & 26,1 \\
\hline Métatarse & 1 & & & & 1 & 4,8 & 2 & 4,3 \\
\hline Métapode & & & & & & & 1 & 2,2 \\
\hline Indéterminé & 1 & & 1 & & 2 & 9,5 & 3 & 6,5 \\
\hline Total & 8 & & 5 & & 21 & 100,0 & 46 & 100,0 \\
\hline
\end{tabular}

Tableau 3

Longueur et largeur maximales des retouchoirs et de leurs zones d'utilisation en mm.

Table 3 Maximal length and breadth of the retouching tools and their utilization zones in $\mathrm{mm}$.

\begin{tabular}{|c|c|c|c|c|c|c|c|c|}
\hline & \multicolumn{4}{|l|}{ A3-A4 } & \multicolumn{4}{|c|}{$\mathrm{A} 5-\mathrm{A} 5+\mathrm{A} 6-\mathrm{A} 6$} \\
\hline & Nombre & Moyenne & Minimum & Maximum & Nombre & Moyenne & Minimum & Maximum \\
\hline Longueur du fragment (LoF, en mm) & 13 & 73,60 & 54 & 112 & 66 & 88,59 & 50 & 166 \\
\hline Largeur du fragment (LaF, en mm) & 13 & 27,40 & 22 & 36 & 66 & 27,17 & 16 & 43 \\
\hline Largeur relative du fragment $(\mathrm{LaF} \times 100 / \mathrm{LoF})$ & 13 & 37,23 & 40,74 & 32,14 & 66 & 30,67 & 32,00 & 25,90 \\
\hline Longueur de l'aire utilisée (LoA, en mm) & 14 & 19,93 & 7 & 90 & 89 & 15,36 & 6 & 44 \\
\hline Largeur de l'aire utilisée (LaA, en mm) & 14 & 8,71 & 6 & 15 & 89 & 9,09 & 2 & 22 \\
\hline Superficie de l'aire utilisée $\left(\mathrm{LoA} \times \mathrm{LaA}\right.$, en $\mathrm{mm}^{2}$ ) & 14 & 173,59 & 42 & 1350 & 89 & 139,62 & 12 & 968 \\
\hline Largeur relative de l'aire utilisée $(\mathrm{LaA} \times 100 / \mathrm{LoA})$ & 14 & 43,70 & 85,71 & 16,67 & 89 & 59,18 & 33,33 & 50,00 \\
\hline
\end{tabular}

même intervalle dimensionnel. Les superficies des zones d'utilisation, cependant, varient selon les complexes technoculturels. Il faut bien entendu tenir compte de l'écart quantitatif important entre les deux corpus, mais nous avons pu noter que les stigmates occupent une plus grande surface sur les pièces uluzziennes. Leur largeur relative, toutefois, est en moyenne supérieure sur les éléments issus du Moustérien final.

Pour toutes les unités stratigraphiques, le poids des supports est assez similaire (Fig. 4), ce qui tend à démontrer qu'ils étaient sélectionnés, voire que les os étaient fracturés en des points spécifiques, pour obtenir des fragments de dimensions et de poids égaux. Un grand nombre de catégories de poids ont été relevées, avec une concentration importante comprise dans l'intervalle de $11 \mathrm{à} 30 \mathrm{~g}$, pour toutes les unités stratigraphiques. Rares sont les pièces qui dépassent ce seuil ; elles proviennent en premier lieu du Moustérien final.

\subsection{Stigmates techniques}

Les retouchoirs sur matières dures animales portent jusqu'à quatre types de stigmates techniques différents :

- empreintes punctiformes-Ce sont les plus fréquentes, toutes unités stratigraphiques confondues (Fig. 5b, d, e, f, h, k, 1). Elles consistent en de petites dépressions, en forme de coin, sub-rectangulaires. Ces empreintes peuvent être plus ou moins profondes et sont souvent accompagnées d'autres traces, comme les stries. Leur concentration varie également et peut cependant être très prononcée (Fig. 5h). Dans certains cas, il est possible d'identifier deux zones d'utilisation différentes, selon l'orientation et la profondeur des stigmates (Fig. 51). Certaines recoupent des stries de raclage, attestant deux phases successives (Fig. 5f, i) ;

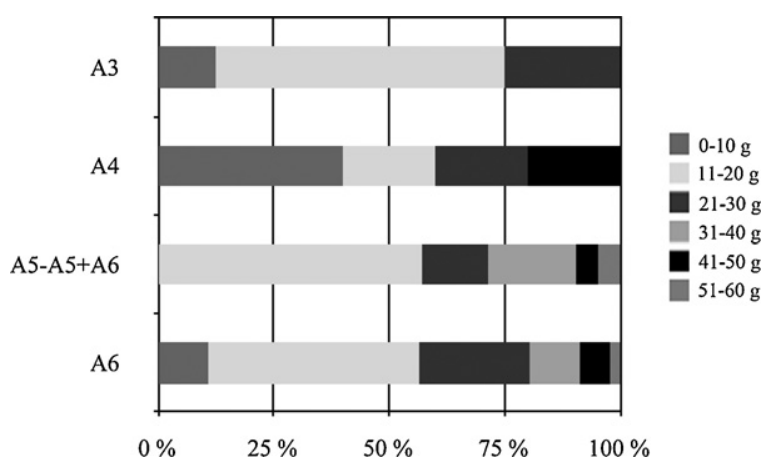

Fig. 4. Pourcentage des catégories de poids par unité stratigraphique; classes de 10 grammes. Les pourcentages pour l'A3 et l'A4 sont donnés à titre indicatif.

Fig. 4. Percentage of weight classes ( 10 grams) per stratigraphic unit. Percentages of A3-A4 are only indicative. 


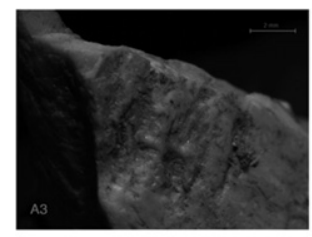

a)

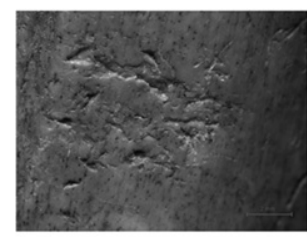

d)

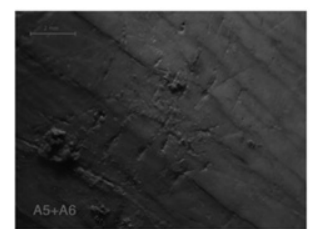

g)

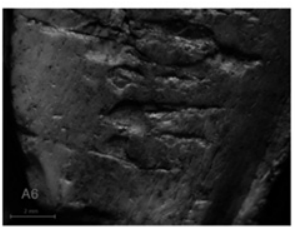

j)

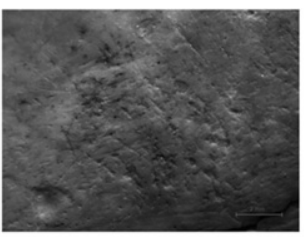

b)

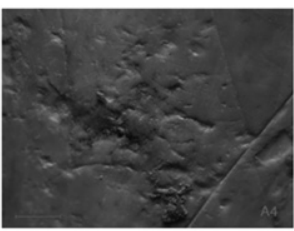

e)

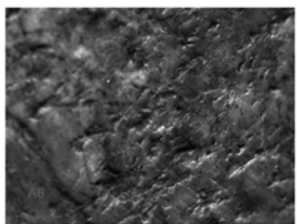

h)

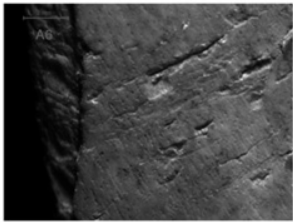

k)

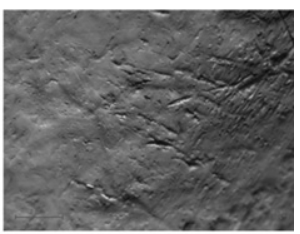

c)

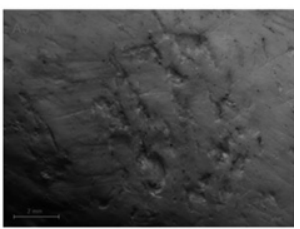

f)

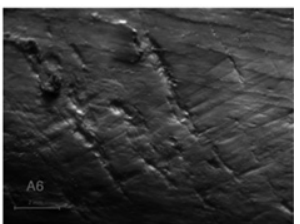

i)

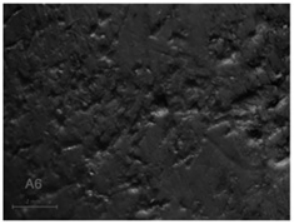

I)

Fig. 5. Typologie des stigmates : a : cupule; b : stries de retouche et empreintes punctiformes; c: empreintes linéaires ; d : empreintes linéaires; e : empreintes linéaires; $\mathrm{f}$ : empreintes punctiformes avec strie de raclage; $\mathrm{g}$ : empreintes linéaires; $\mathrm{h}$ : empreintes punctiformes très concentrées; i: empreintes linéaires et punctiformes avec stries de raclage; $\mathrm{j}$ : empreintes linéaires et cupules; $\mathrm{k}$ : empreintes linéaires et punctiformes; 1 : empreintes punctiformes, intersection entre deux zones d'utilisation.

Fig. 5. Types of stigmata: a: notch; b: strias and punctiform impressions; c: linear impressions; d: linear impressions; e: linear impressions; f: punctiform impressions with scraping strias; g: linear impressions; h: very dense punctiform impressions; i: linear and punctiform impressions with scraping strias; $\mathrm{j}$ : linear impressions and wells; k: linear and punctiform impressions; l: punctiform impressions, intersection with two utilization zones.

- empreintes linéaires - Leur morphologie est similaire à des stries mais elles sont plus larges et plus profondes (Fig. 5c, e, g, i, j, k). Cette morphologie peut cependant varier passablement. Ces empreintes sont généralement associées à d'autres types de stigmates ;

- stries dues à la retouche - Cette typologie est illustrée sur la Fig. 5b. Les stries sont généralement fines et courtes et, dans la plupart des cas, associées aux empreintes linéaires ;

- cupules - Elles consistent en de gros enlèvements de matière, dus notamment à des coups répétés sur la surface concernée (Fig. $5 \mathrm{a}, \mathrm{j}$ ). Elles peuvent présenter plusieurs morphologies, suivant la force utilisée, le nombre d'éclats qui les ont créées et le type de stigmate majoritaire.

Les retouchoirs peuvent contenir jusqu'à trois zones d'utilisation (Fig. 6), bien que, dans la très grande majorité

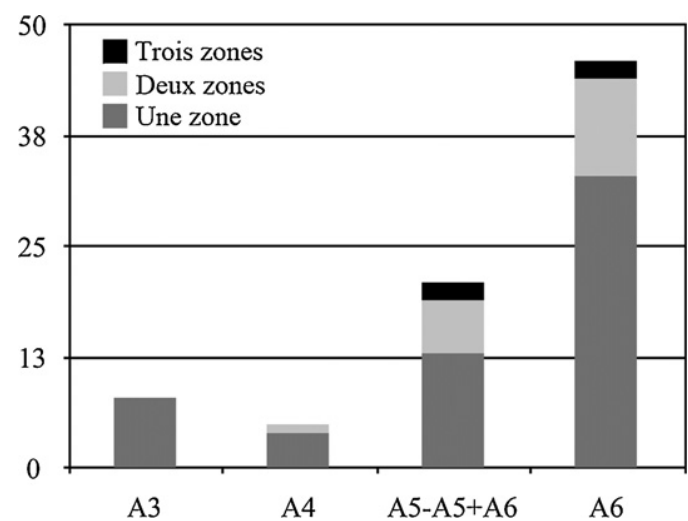

Fig. 6. Nombre des zones d'utilisation par unité stratigraphique. Fig. 6. Number of utilization zones per stratigraphic unit. 


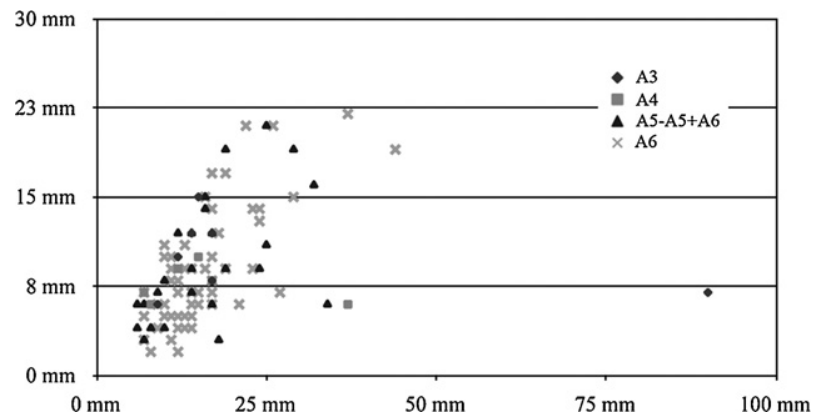

Fig. 7. Longueur (abscisse) et largeur (ordonnée) en mm des zones d'utilisation, par unité stratigraphique.

Fig. 7. Length (abscissa) and width (ordinate) in $\mathrm{mm}$ of the utilization zones, per stratigraphic unit.

des cas, les fragments n'en attestent qu'une. Comme illustré plus haut, les surfaces utilisées peuvent parfois se superposer et s'entrecroiser. Dans le cas de retouchoirs à plusieurs zones, nous avons relevé la prépondérance systématique d'une surface d'utilisation sur l'autre ou les autres.

La superficie des zones d'utilisation, quant à elle, reste relativement homogène, comme en témoigne la Fig. 7. Cependant, le Tableau 3 montre que les aires utilisées sont généralement plus importantes dans les couches uluzziennes.

\section{Discussion}

Les sites contenant des retouchoirs pour cette période chronologique montrent clairement une préférence pour les grands herbivores (Armand et Delagnes, 1998 ; Castel et al., 2003; Mozota Holgueras, 2009; Schwab, 2002 ; Tartar, 2009; Vincent, 1993). De même, les segments anatomiques choisis sont généralement similaires: fragments de diaphyses d'os longs. Cependant, de nombreux retouchoirs ont été identifiés également sur les épiphyses, alors qu'à Fumane, il n'en a été trouvé aucun. Cela peut s'expliquer par la fragmentation des os (Peresani et al., 2011b ; Romandini, 2007-2008; Tagliacozzo et al., à paraître) ou la sous-représentation de ces portions anatomiques. En règle générale, au sein des niveaux analysés, le taux de fragmentation des os est très élevé : plus de $90 \%$ du nombre de restes se trouve dans la plus petite classe dimensionnelle (longueur maximale $3 \mathrm{~cm}$ ). Les os calcinés comprennent beaucoup de petits fragments de diaphyses et d'os spongieux. Ces données indiquent l'utilisation de l'os comme combustible (Costamagno et Théry-Parisot, 2005) pour les feux qui ont été allumés dans la grotte.

Les retouchoirs comportent de nombreux points communs entre les différents niveaux. En effet, plusieurs critères, tels que l'espèce, le segment anatomique ou le poids attestent une homogénéité générale. La recherche de supports en partie standardisés rejoindrait les conclusions de Mozota Holgueras (2009), à la suite d'une série d'expérimentations montrant que les diaphyses osseuses avaient probablement fait l'objet d'une sélection, quant au poids et aux dimensions des supports. Selon lui, les os longs étaient fracturés selon un modèle précis, qui permettait d'obtenir des supports adéquats pour la retouche. Ces résultats, bien qu'un peu trop définitifs à nos yeux, semblent corroborés, au moins en partie, par les données obtenues sur les fragments osseux de la grotte de Fumane. Nous ne pouvons cependant pas exclure que les os longs ont été fracturés sans relation particulière avec l'obtention de retouchoirs et que les éléments idoines à la retouche ont fait ultérieurement l'objet d'une sélection casuelle.

Les surfaces d'utilisation présentent des disparités en fonction des complexes technoculturels. Dans leur article, Verna et d'Errico (2011) rapportent des largeurs relatives de ces aires nettement supérieures aux valeurs obtenues pour la grotte de Fumane. Dans la mesure où il s'agit d'une étude qui a porté sur des retouchoirs issus des niveaux à débitage Quina, il est possible que les zones d'utilisation des retouchoirs aient été plus larges, du fait de la différence de complexe technoculturel. En effet, l'Uluzzien, qui requiert plus de précision lors de la retouche puisque les outils lithiques sont plus petits, a des surfaces d'utilisation relativement nettement moins larges que les autres.

\section{Conclusions}

Les stigmates techniques des retouchoirs en matières dures animales du Moustérien final et de l'Uluzzien de la grotte de Fumane n'attestent pas des différences notables entre les deux complexes culturels. Malgré les dissemblances au niveau de l'industrie lithique, en particulier en ce qui concerne la retouche, les coups portés à l'aide d'un retouchoir ont laissé des traces similaires et dans des proportions généralement proches. Ces retouchoirs rejoignent en grande partie les pièces mises au jour dans d'autres sites de la région, proches également d'un point de vue chronologique, comme Riparo Tagliente (Leonardi, 1979; Thun Hohenstein, 2006), Grotta della Ghiacciaia (Bertola et al., 1999) et Grotta Maggiore di San Bernardino (Giacobini et Malerba, 1996), dont les études ne sont toutefois pas encore terminées. En revanche, l'Uluzzien innove par rapport au Moustérien sous-jacent par l'utilisation de segments anatomiques d'ours brun, pour lesquelles l'hypothèse de la disponibilité sur le site peut être soutenue.

\section{Remerciements}

Les recherches sur la grotte de Fumane ont pu être conduites, grâce au soutien du Superintendant pour le Patrimoine archéologique de la Vénétie, la Région de la 
Vénétie, la Fondation Cariverona, la Comunità Montana della Lessinia, la municipalité de Fumane et les compagnies privées. La plupart des données archéozoologiques obtenues dans cette étude sont issues du mémoire de Master de M. Romandini (2007-2008) et de M.De March (2009-2010). Nous remercions A. Tagliacozzo du Musée Pigorini à Rome de nous avoir permis d'utiliser la collection ostéologique de comparaison. Texte français révisé par Jean-Pierre Chadelle. M.P. a dirigé les recherches, C.J. a analysé les retouchoirs et M. R. l'archéozoologie. Tous les auteurs ont contribué à la rédaction du texte.

\section{References}

Armand, D., Delagnes, A., 1998. Les retouchoirs en os d'Artenac (couche 6c): perspectives archéozoologiques, taphonomiques et expérimentales, Economie Préhistorique. In: Brugal, J. Ph., Meignen, L. Patou-Mathys, M. (Eds.), Les comportements de subsistance au Paléolithique, $x_{I I}{ }^{\mathrm{e}}$ Rencontres Internationales d'Archéologie et d'Histoire d'Antibes, APDCA, Sophia Antipolis, pp. 205-214.

Auguste, P., 2002. Fiche éclats diaphysaires du Paléolithique moyen: Biache-Saint-Vaast (Pas-de-Calais) et Kulna (Moravie, République tchèque). In: Patou-Mathis, M. (Ed.), Retouchoirs, compresseurs, percuteurs. Os à impressions et éraillures. Société Préhistorique Française, Paris, pp. 39-57, X.

Bertola, S., Peresani, M., Peretto, C., Thun-Hohenstein, U., 1999. Le site Paléolithique moyen de la Grotta della Ghiacciaia (Préalpes de Vénétie, Italie du Nord). L'Anthropologie 103, 377-390.

Binford, L.R., 1981. Bones: ancient men and modern myths. Academic Press Inc.

Bordes, F., 1961. Typologie du Paléolithique ancien et moyen. Université de Bordeaux, Institut de Préhistoire, Bordeaux, 102 p.

Bourlon, M., 1916. Nouvelles découvertes à Laugerie-Basse. Rabots, os utilisés, œuvres d'art. L'Anthropologie 27, 10.

Broglio, A., Dalmeri, G. (Eds.). 2005. Pitture paleolitiche nelle Prealpi Venete - Grotta di Fumane e Riparo Dalmeri. Preist. Alp., 12-22.

Broglio, A., De Stefani, M., Tagliacozzo, A., Gurioli, F., Facciolo, A., 2006. Aurignacian dwelling structures, hunting strategies and seasonality in the Fumane Cave (Lessini Mountains). In: Vasil'ev, S.A., Popov, V.V., Anikovich, M.V., Praslov, N.D., Sinitsyn, A.A., Hoffecker, J.F. (Eds.), Kostenki and the Early Upper Paleolithic of Eurasia: general trends, local developments. Nestor-Historia, Saint-Petersbourg, pp. 263-268.

Cassoli, PF., Tagliacozzo, A. 1994. Considerazioni paleontologiche, paleoecologiche e archeozoologiche sui macromammiferi e gli uccelli dei livelli del Pleistocene superiore del Riparo di Fumane (VR) (scavi 1988-91). Bull. Mus. Civ. St. Nat. Verona 18, 349-445.

Castel, J.C., Chauvière, F.X., Madelaine, S., 2003. Sur os et sur dents : les "retouchoirs » aurignaciens de La Ferrassie (Savignac-de-Miremont, Dordogne). Paléo 15, 29-50.

Costamagno, S., Théry-Parisot, I., 2005. Propriétés combustibles des ossements : données expérimentales et réflexions archéologiques sur leur emploi dans les sites paléolithiques. Gallia Préhist. 47, 235-254.

D'Errico, F., Borgia, V., Ronchitelli, A. (sous presse). Uluzzian bone technology and its implications for the origin of the behavioural modernity. Quat. Int. doi:10.1016/j.quaint.2011.03.039.

De March, M., 2009-2010. Studio archeozoologico dell'insieme faunistico dei livelli del Musteriano finale A5+A6e A6 della Grotta di Fumane (Monti Lessini, VR), Mémoire de Master, Université de Ferrara, Ferrara, $274 \mathrm{p}$.

Giacobini, G., Malerba, G., 1996. Les retouchoirs sur éclats diaphysaires du Paléolithique moyen et supérieur de trois sites de l'Italie nordorientale (Grotte de San Bernardino, Abri de Fumane et Abri Tagliente) In: Facchini, F., Palma di Cesnola, A., Piperno, M., Peretto, C. (Eds.), XIII ${ }^{\mathrm{e}}$ Congrès de l'Union Internationale des Sciences Préhistoriques et Protohistoriques (UISPP). Forlì ABACO, pp. 167-171.

Gruet, M., 1947. Présence d'industries aurignaciennes dans le Sud du Massif Armoricain. Bull. Soc. Pr. Ehist. Fr. 4, 182-191.

Henri-Martin, L., 1906. Maillets ou enclumes en os provenant de la couche moustérienne de la Quina (Charente). Bull. Soc. Pr. Ehist. Fr. 3, 155-162.

Higham, T., Brock, F., Peresani, M., Broglio, A., Wood, R., Douka, K., 2009. Problems with radiocarbon dating the Middle to Upper Palaeolithic transition in Italy. Quat. Sci. Rev. 28, 1257-1267.

Leonardi, P., 1979. Una serie di ritoccatoi prevalentemente musteriani del Riparo Tagliente in Valpantena presso Verona. Preist. Alp. 15, 7-15.
Mallye, J.-B., Mourre, V., Thiébaut, C., Costamagno, S., 2008. Retouchoirs et autres " os à impressions et éraillures " : caractérisation et fonctionnement. In: Culture, M.f.d.l. (Ed.), PCR « Des Traces et des Hommes ». Université de Toulouse, Toulouse, pp. 213-230.

Mozota Holgueras, M., 2007. El hueso como materia prima: Las industrias óseas del final del Musteriense en la Región Cantábrica. Los niveles B-C-D de Axlor (Dima, Bizkaia). Mémoire de Master, Universidad de Cantabria, Santander, $100 \mathrm{p}$.

Mozota Holgueras M., 2007. Estudio tafonómico y tecnológico de un útil doble "cincel/retocador", proveniente del nivel C (Musteriense) de Axlor-Dima, Bizkaia. Zephyrus 60, 207-214.

Mozota Holgueras, M., 2009. El utillaje óseo musteriense del nivel « D » de Axlor (Dima, Vizcaya): análisis de la cadena operativa. Trab. Prehist. 66, 27-46.

Oliva, M., 1995. Le Szélétien de Tchécoslovaquie: industrie lithique et répartition géographique. Paléo Suppl., 83-90.

Patou-Mathis, M. (Ed.), 2002. Retouchoirs, compresseurs, percuteurs... Os à impressions et éraillures. Société Préhistorique Française, Paris, p. 136.

Patou-Mathis, M., Schwab, C., 2002. Fiche générale. In: Patou-Mathis, M. (Ed.), Retouchoirs, compresseurs, percuteurs. Os à impressions et éraillures. Société Préhistorique Française, Paris, X, pp. 11-19.

Peresani, M., 2008. A New Cultural Frontier for the Last Neanderthals: the Uluzzian in northern Italy. Curr. Anthr. 49, 725-731.

Peresani, M., 2012. Fifty thousand years of flint knapping and tool shaping across the Mousterian and Uluzzian sequence of Fumane cave. In: Carbonnell, E., Gema, M., Vaquero, M. (Eds.), The Neanderthal Home: Spatial and Social Behaviours. Quat. Int. 247, $125-150$

Peresani, M., Cremaschi, M., Ferraro, F., Falguères, C., Bahain, J.-J., Gruppioni, G., Sibilia, E., Quarta, G., Calcagnile, L., Dolo, J.-M., 2008. Age of the final Middle Palaeolithic and Uluzzian levels at Fumane Cave, northern Italy, using ${ }^{14} \mathrm{C}$, ESR, ${ }^{234} \mathrm{U} /{ }^{230} \mathrm{Th}$ and thermoluminescence methods. J. Archaeol. Sc. 35, 2986-2996.

Peresani, M., Fiore, I., Gala, M., Romandini, M., Tagliacozzo, A., 2011a. Late Neandertals and the intentional removal of feathers as evidenced from bird bone taphonomy at Fumane Cave 44 ky B.P., Italy. Pr. Nat. Acad. Sc. $108,3888-3893$.

Peresani, M., Chravzez, J., Danti, A., De March, M., Duches, R., Gurioli, F., Muratori, S., Romandini, M., Tagliacozzo, A., Trombino, L., 2011b. Fire-places frequentations and the environmental setting of the final Mousterian at Grotta di Fumane: a report from the 2006-2008 research. Quartär 58, 131-151.

Roberts, M.B., Parfitt, S.A., 1999. Boxgrove: a Middle Pleistocene Hominid Site at Eartham Quarry, Boxgrove, West Sussex. English Heritage, London, $456 \mathrm{p}$.

Romandini, M., 2007-2008. Sfruttamento del cervo e della volpe nei livelli uluzziani della Grotta di Fumane (Monti Lessini VR), Mémoire de Master, Universitè de Ferrara, Ferrara, 232 p.

Schwab, C., 2002. Fiche éclats diaphysaires du Paléolithique moyen et supérieur: la grotte d'Isturitz (Pyrénées-Atlantiques). In: PatouMathis, M. (Ed.), Retouchoirs, compresseurs, percuteurs. Os à impressions et éraillures. Société Préhistorique Française, Paris, X, pp. 59-73.

Simán, K., 1995. La grotte Szeleta et le Szélétien. Paléo Suppl.1, 37-43.

Slimak, L., 2007. Le Néronien et la structure historique du basculement du Paléolithique moyen au Paléolithique supérieur en France méditerranéenne. C. R. Palevol. 6, 301-309.

Tagliacozzo, A., Romandini, M., Gala, M., Fiore, I., Peresani, M., à paraître. Animal exploitation strategies during the Uluzzian at Grotta di Fumane (Verona). In: Delson, E., Sargis, E. (Eds.), Vertebrate Paleobiology and Paleoanthropology. International Conferences of Archaeozoology, Paris 2010, Springer, New York.

Tartar, E., 2009. De l'os à l'outil. Caractérisation technique, économique et sociale de l'utilisation de l'os à l'Aurignacien ancien - Etude de trois sites : l'Abri Castanet (secteurs nord et sud), Brassempouy (Grotte des Hyènes et Abri Dubalen) et Gatzarria, PhD Thesis, Universitè Paris I - Panthéon-Sorbonne, Paris, 308 p.

Thun Hohenstein, U., 2006. Strategie di sussistenza adottate dai Neandertaliani nel sito di Riparo Tagliente (Prealpi venete). In: Tecchiati, U. (Ed.), Archaeozoological studies in honour of Alfredo Riedel, Bolzano, pp. 31-38.

Valensi, P., Psathi, E., 2004. Faunal Exploitation during the Middle Palaeolithic in south-eastern France and north-western Italy. Int. J. Osteoarchaeol. 14 (3-4), 256-272.

Verna, C., d'Errico, F., 2011. The earliest evidence for the use of human bone as a tool. J. Hum. Evol. 60, 145-157.

Vincent, A., 1993. L'outillage osseux au Paléolithique moyen : Une nouvelle approche, Phd Thesis, Université Paris-X, Paris, 317 p. 